\title{
AN ADVANCED LEVEL SET METHOD BASED ON BREGMAN DIVERGENCE FOR INHOMOGENEOUS IMAGE SEGMENTATION
}

\author{
DAMING SHI ${ }^{1}$,MEILU ZHU ${ }^{1}$,YONGQIANG ZHANG ${ }^{2}$,FENG TIAN ${ }^{3}$ \\ ${ }^{1}$ School of Computer Science and Software Engineering, Shenzhen University, China \\ ${ }^{2}$ School of Computer Science and and technology, Harbin Institute of Technology, China \\ ${ }^{3}$ School of Design, Engineering and Computing, Bournemouth University, BH12 5BB, UK
}

\begin{abstract}
:
Intensity inhomogeneity often occurs in real images. Local information based level set methods are comparatively effective in segmenting image with inhomogeneous intensity. However, in practice, these models suffer from local minima and high computational cost. In this paper, a novel region-based level set method based on Bregman divergence and local binary fitting, hereafter referred to as Bregman- $\mathrm{LBF}$, is proposed for image segmentation. The proposed method utilizes global and local information to formulate a new energy function. The Bregman-LBF model enjoys the following advantages: (1) Bregman-LBF outperforms the piece-wise constant(PC) model in handling intensity inhomogeneity. (2) Bregman-LBF is more effective than the local binary fitting (LBF) model and more robust than the global and local intensity fitting (GLIF) model. The relationship between the Bregman-LBF model and the existing models, e.g. the Chan-Vese(CV) model, is discussed. The experiments conducted on synthetic and benchmark image datasets have shown that the proposed Bregman-LBF outperforms the piece-wise constan$t$ (PC) model in handling intensity inhomogeneity. The experimental results have also shown that the Bregman-LBF is more effective than the local binary fitting (LBF) model and more robust than the global and local intensity fitting (GLIF) model.
\end{abstract}

\section{Keywords:}

Level set; Image segmentation; Bregman divergence

\section{Introduction}

Image segmentation is important for visual information analysis processes, such as object detection and scene understanding. Even though many methods have been developed to solve this problem, image segmentation still is a research challenge. The Active Contour Model (ACMs, also called snakes or de-
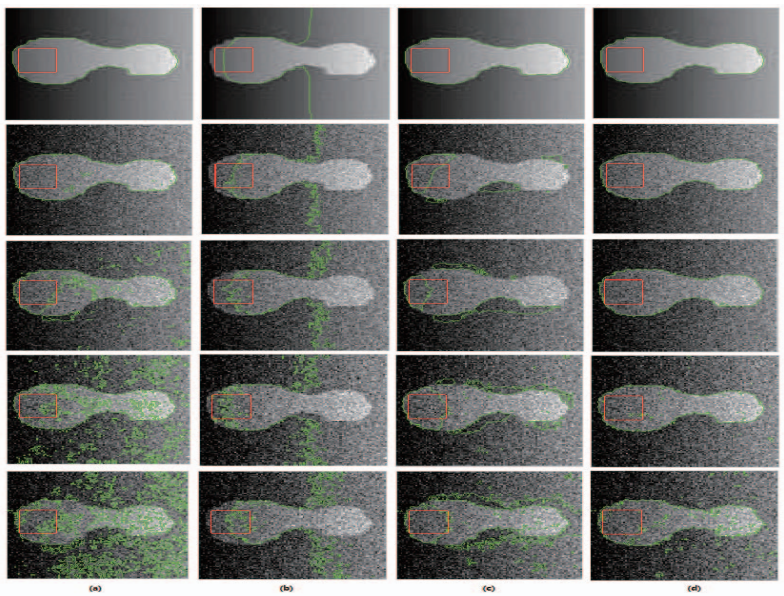

FIGURE 1. Segmentation results of the Bregman-LBF model in different noise image conditions. The red contour is the initial contour, the green contours are the segmentation results. (The level of Gaussian noise correspond to each column are mean $\mu=0$, variance $\sigma^{2}=$ $0,0.001 * 255^{2}, 0.002 * 255^{2}, 0.003 * 255^{2}, 0.004 * 255^{2}$, respective1y).

formable models) initially proposed by [3], is one of the most successful methods for image segmentation. Active contour models can be generally categorized into two classes according to the nature of constraints: the edge-based models ([3], and references therein); and the region-based models ([2]; [9][4][13], and references therein). An outstanding characteristic of edgebased methods and region-based methods is that they partition an image into sub-regions with closed and smooth boundaries. However, the edge-based methods have some drawbacks. For example, they are sensitive to image noise, weak edges, initial placement of the curve, difficulty in handling topological changes, and its dependency of parameterization. Compared with the edge-based methods, region-based methods which u- 
tilize the PDE-based level set method introduced by [8] represent the curves as a zero level set of a higher dimensional function. The level set method can naturally represent the contours of complex topology and deal with topological changes (contour splitting and merging). Thus, region-based method$s$ avoid the drawbacks of difficulty in coping with topological changes and dependency of parameterization of the edge-based methods. Moreover, region-based methods are more robust to image noise and the initial position of zero level set. They also significantly improve the segmentation accuracy for images with weak boundaries.

In the Mumford-Shah model [7], an image is decomposed into some sub-regions, each of which is approximated by a smooth function. The optimal partition of the image can be derived by minimizing the Mumford-Shah functional. Based on the Mumford-Shah functional for segmentation, [2] developed the easily handled Chan-Vese (CV) model. The most remarkable feature of the $\mathrm{CV}$ model is that the computational cost is low with fast speed. The basic assumption which promises the $\mathrm{CV}$ model working well is that the intensity of each region of image is statistically uniform; otherwise, the CV model cannot correctly segment the image with inhomogeneous intensity and the details of the object. To solve this issue, by assuming that the intensities in a relatively small local region are separable, [12] and [11] respectively proposed two similar region-based active contour models, widely known as piecewise smooth $(\mathrm{P}-$ $\mathrm{S})$ models, These models improve the capability of addressing the intensity inhomogeneity. However, the piecewise smooth models are computationally expensive.

The general region-based image segmentation method typically relies on a specific region descriptor (e.g., intensity mean or a Gaussian distribution) of intensities in each region to be segmented. However, intensity inhomogeneity often leads to overlap between the distribution of the intensities in each subregion of the image. It is difficult to give such a regional descriptor for images with intensity inhomogeneity. To solve the problem of intensity inhomogeneity, researcher use the local statistical information of intensity, e.g. the local mean of intensity, the local intensity distribution or the histogram of the intensity, to approximates the image for improving segmentation accuracy ([9][17]). The local binary fitting (LBF) model proposed by [4] and the local Gaussian distribution fitting (LGD) model proposed by [13] are two such examples. Even though these methods can commendably cope with image inhomogeneity, the obvious limitation of this kind of methods is the sensitivity to the placement of initial contour and high computational expense. To overcome the problem of sensitivity to the initial placement of the local information based methods,
[15] proposed a local Chan-Vese(LCV) model which can utilize both global image information and local image information for image segmentation. The LCV improves the segmentation result of the inhomogeneous image to some certain degree and is robust to the placement of initial contour, but [6] pointed out that the LCV model is also a CV model, to some extent. It is difficult for the LCV model to satisfactorily segment the image with intensity inhomogeneity.[14] proposed a local binary fitting incorporated with local order energy, this model utilize the local constrain to improve the stability. The combination of the global and the local information is in principle in favor in solving both the initial sensitivity and the intensity inhomogeneity problems [16]. However, the current models all utilize the $L^{2}-$ norm to measure the global information while none uses the Bregman divergence, which can be considered as a data-dependent weighted $L^{2}-$ norm, Compared with the $L^{2}-$ norm, the Bregman divergence apply multi-level information to measure the global information, especially, the datadependent weighted term can be consider as the prior information. Then the data-dependent weighted $L^{2}-$ norm, takes advantage of multi-level information of image which can assist boosting stability of the image segmentation model, especially, the robustness to initial placement.

In this paper, an advanced region-based method based on Bregman divergence and local binary fitting is proposed. The Bregman divergence is utilized to effectively measure global information, Applying the Taylor expansion, we can derive that the Bregman divergence can be approximated by a datadependent weighted $L^{2}-$ norm, and the data-related weighted term can be considered as the prior information which can accelerate the contour evolution. Hence the Bregman divergence can boost stability and accelerate the curve evolution. Simultaneously, local data information is used to improve the capability of handling the intensity inhomogeneity.

The remainder of this paper is organized as follows. In Section 2, we propose our model and illustrate its advantages. Then we validate our model by experiments on synthetic images, medical images, and real world images in Section 3. The conclusions are drawn in Section 4.

\section{Bregman Divergence Incorporated with Local Bi- nary Fitting}

In this section, we will propose our new region-based Bregman-LBF model, which is based on Bregman divergence ([1][10]) and local binary fitting. 

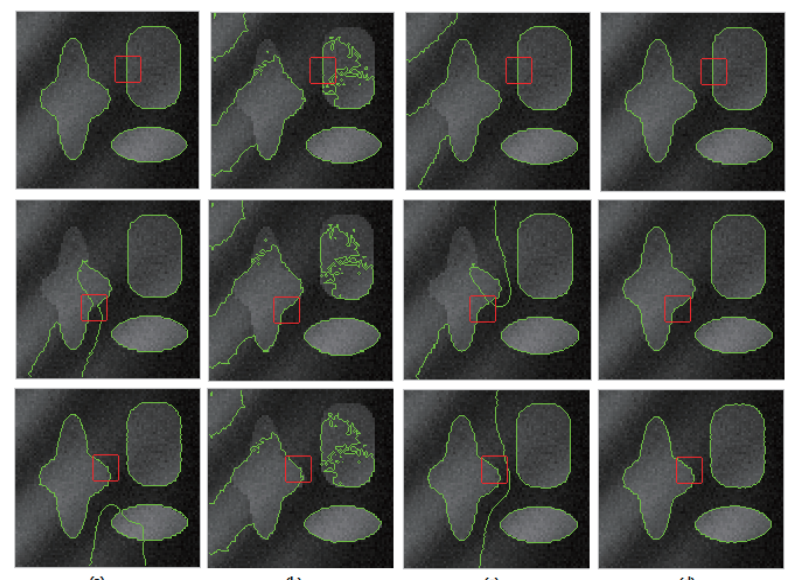

(a)

(b)
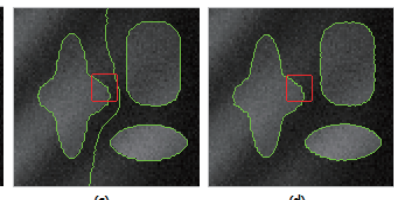

(c)

FIGURE 2. Segmentation Results of the LBF model, the LCV model, the LGIF model and the Bregman-LBF model with different initial positions. The red contour is the initial contour, while the green contour is the segmentation results. Columns (a), (b), (c), (d) are the segmentation results of the LBF model, the LCV model, the LGIF model and the Bregman-LBF model, respectively.

\subsection{Bregman Divergence for Energy Measure}

To implement the segmentation of inhomogeneity image and solve the sensitivity to initial placement, multi-level information is utilized to formulate the proposed model which is defined as the following minimization problem:

$$
\begin{aligned}
& E^{\text {Bregman-LBF}}\left(\boldsymbol{C}, c_{1}, c_{2}, f_{1}, f_{2}\right) \\
& =w E^{B}\left(\boldsymbol{C}, c_{1}, c_{2}\right)+(1-w) E^{L}\left(\boldsymbol{C}, f_{1}, f_{2}\right)+E^{R}(\boldsymbol{C})
\end{aligned}
$$

where

$$
\begin{aligned}
& E^{B}\left(\boldsymbol{C}, c_{1}, c_{2}\right)=\lambda_{1} \int_{i n(\boldsymbol{C})} B_{\varphi(x)=x^{2 \alpha}}\left(c_{1} \| I_{\boldsymbol{x}}\right) d \boldsymbol{x} \\
& +\lambda_{2} \int_{\text {out }(\boldsymbol{C})} B_{\varphi(x)=x^{2 \beta}}\left(c_{2} \| I_{\boldsymbol{x}}\right) d \boldsymbol{x} \\
& E^{L}\left(\boldsymbol{C}, f_{1}, f_{2}\right)=\int_{\Omega} E_{\boldsymbol{x}}\left(\boldsymbol{C}, f_{1}(\boldsymbol{x}), f_{2}(\boldsymbol{x})\right) d \boldsymbol{x} \\
& E^{R}(\boldsymbol{C})=\mu \text { Length }(\boldsymbol{C})
\end{aligned}
$$

with $\alpha, \beta, \lambda_{1}, \lambda_{2}$ are positive constants, and $\alpha \geq 1 \geq \beta \geq 0$, $C$ is the curve, $c_{1}, c_{2}$ are the mean intensities inside and outside $C$, respectively. $w$ is the weight factor to balance the global information energy term $E^{B}$ and the local information energy term $E^{L} \cdot B_{\varphi(x)=x^{2 \alpha}}\left(c_{1} \| I_{\boldsymbol{x}}\right)$ and $B_{\varphi(x)=x^{2 \beta}}\left(c_{2} \| I_{\boldsymbol{x}}\right)$ are the

Bregman divergence corresponding to the point pair $\left(c_{1}, I_{\boldsymbol{x}}\right)$ and $\left(c_{2}, I_{\boldsymbol{x}}\right)$, respectively. $I_{\boldsymbol{x}}$ is the intensity at the point $\boldsymbol{x}$. The local information energy term is defined as follow.

$$
\begin{aligned}
& E_{\boldsymbol{x}}\left(\boldsymbol{C}, f_{1}(\boldsymbol{x}), f_{2}(\boldsymbol{x})\right) \\
& =\lambda_{1} \int_{i n(\boldsymbol{C})} g_{k}(\boldsymbol{x}-\boldsymbol{y})\left(I_{\boldsymbol{y}}-f_{1}(\boldsymbol{x})\right)^{2} d \boldsymbol{y} \\
& +\lambda_{2} \int_{\text {out }(\boldsymbol{C})} g_{k}(\boldsymbol{x}-\boldsymbol{y})\left(I_{\boldsymbol{y}}-f_{2}(\boldsymbol{x})\right)^{2} d \boldsymbol{y}
\end{aligned}
$$

For the energy term $E^{B}$, according to the Taylor's expansion, we can get the following equation:

$$
\begin{aligned}
& B_{\varphi(x)=x^{2 \alpha}}\left(c_{1} \| I_{\boldsymbol{x}}\right)=\alpha_{1} I_{\boldsymbol{x}}^{2 \alpha-2}\left(I_{\boldsymbol{x}}-c_{1}\right)^{2}+R_{1} \\
& B_{\varphi(x)=x^{2 \beta}}\left(c_{2} \| I_{\boldsymbol{x}}\right)=\beta_{1} I_{\boldsymbol{x}}^{2 \beta-2}\left(I_{\boldsymbol{x}}-c_{2}\right)^{2}+R_{2}
\end{aligned}
$$

where $R_{1}=o\left(\left|I_{\boldsymbol{x}}-c_{1}\right|^{3}\right), R_{2}=o\left(\left|I_{\boldsymbol{x}}-c_{2}\right|^{3}\right)$ are the thirdorder of the Taylor expanded remaining terms, and $\alpha_{1}=2 \alpha^{2}-$ $\alpha, \beta_{1}=2 \beta^{2}-\beta$ are two coefficients.

Only the zeroth-, the first- and the second- order the Taylor terms are considered in this research. Hence, the Bregman divergence can be considered as the data-dependent weighted $L^{2}-$ norm. Then the energy term $E^{B}$ is equivalent to:

$$
\begin{aligned}
E^{B} & =\lambda_{1} \int_{i n(\boldsymbol{C})} \alpha_{1} I_{\boldsymbol{x}}^{2 \alpha-2}\left(I_{\boldsymbol{x}}-c_{1}\right)^{2} d \boldsymbol{x} \\
& +\lambda_{2} \int_{\text {out }(\boldsymbol{C})} \beta_{1} I_{\boldsymbol{x}}^{2 \beta-2}\left(I_{\boldsymbol{x}}-c_{2}\right)^{2} d \boldsymbol{x}
\end{aligned}
$$

To avoid the time-consuming re-initialization and preserve the regularity of the level set function, a penalization term ([5]) is introduced to our active contour model:

$$
E^{P}(\phi)=v \int_{\Omega} \frac{1}{2}(|\nabla \phi(\boldsymbol{x})|-1)^{2} d \boldsymbol{x}
$$

where $v$ is a positive constant, and in the most cases, $v=1$. Hence, we can obtain the following equation:

$$
\begin{aligned}
& E^{\text {Bregman-LBF }}\left(\phi, c_{1}, c_{2}, f_{1}, f_{2}\right) \\
= & \lambda_{1} \int_{\Omega}\left(e_{1}+e_{3}\right) H_{\varepsilon}(\phi(\boldsymbol{x})) d \boldsymbol{x} \\
& +\lambda_{2} \int_{\Omega}\left(e_{2}+e_{4}\right)\left(1-H_{\varepsilon}(\phi(\boldsymbol{x}))\right) d \boldsymbol{x} \\
& +\mu \int_{\Omega} \delta_{\varepsilon}(\phi(\boldsymbol{x})) d \boldsymbol{x}+v \int_{\Omega} \frac{1}{2}(|\nabla \phi(\boldsymbol{x})|-1)^{2} d \boldsymbol{x}
\end{aligned}
$$


where $\phi(\boldsymbol{x})$ is the level set function, $H_{\varepsilon}(\phi(\boldsymbol{x}))$ and $\delta_{\varepsilon}(\phi(\boldsymbol{x}))$ are Heaviside function and Dirac function, respectively,

$$
\begin{aligned}
& e_{1}(\boldsymbol{x})=(1-w) \int_{\Omega} g_{k}(\boldsymbol{y}-\boldsymbol{x})\left|I_{\boldsymbol{x}}-f_{1}(\boldsymbol{y})\right|^{2} d \boldsymbol{y} \\
& e_{2}(\boldsymbol{x})=(1-w) \int_{\Omega} g_{k}(\boldsymbol{y}-\boldsymbol{x})\left|I_{\boldsymbol{x}}-f_{2}(\boldsymbol{y})\right|^{2} d \boldsymbol{y} \\
& e_{3}(\boldsymbol{x})=w \alpha_{1} I_{\boldsymbol{x}}^{2 \alpha-2}\left(I_{\boldsymbol{x}}-c_{1}\right)^{2} \\
& e_{4}(\boldsymbol{x})=w \beta_{1} I_{\boldsymbol{x}}^{2 \beta-2}\left(I_{\boldsymbol{x}}-c_{2}\right)^{2} \\
& H_{\varepsilon}(x)=\frac{1}{2}\left[1+\frac{2}{\pi} \arctan \left(\frac{x}{\epsilon}\right)\right] \\
& \delta_{\varepsilon}(x)=H^{\prime}{ }_{\varepsilon}(x)=\frac{1}{\pi} \frac{\varepsilon}{\varepsilon^{2}+x^{2}}
\end{aligned}
$$

where $\varepsilon$ is a small positive constant. Let $\epsilon \rightarrow 0, H_{\epsilon}(x)$ and $\delta_{\epsilon}(x)$ s.t.

$$
\begin{aligned}
& \lim _{\epsilon \rightarrow 0} H_{\epsilon}(x)=H(x)= \begin{cases}1, & x \geq 0 \\
0, & \text { otherwise }\end{cases} \\
& \lim _{\epsilon \rightarrow 0} \delta_{\epsilon}(x)= \begin{cases}1, & x=0 \\
0, & \text { otherwise }\end{cases}
\end{aligned}
$$

The value of $\epsilon$ relate to the speed of the contour evolution and the accuracy, but to make the level set evolution fast, typically, $\epsilon=1 . \alpha_{1}, \beta_{1}$ are same as that in the Equation (5).
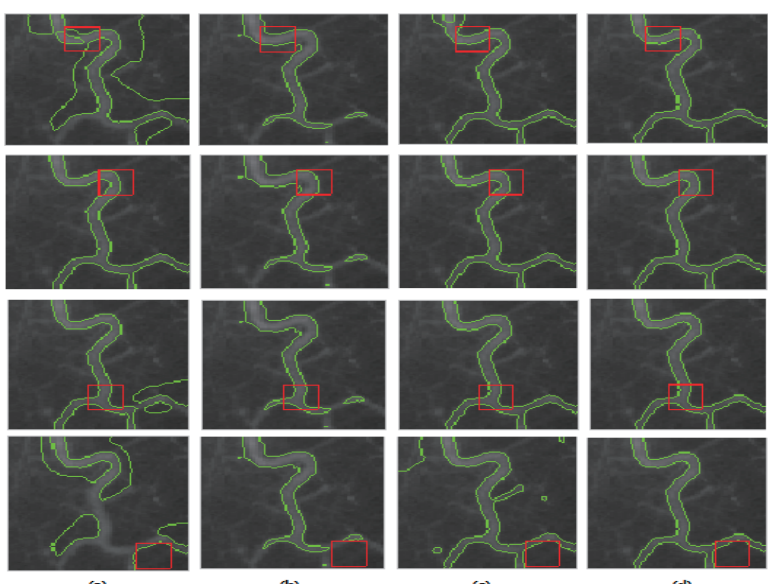

(b)

(c)

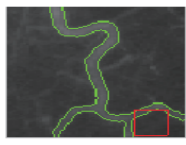

(d)

FIGURE 3. Segmentation Results of the LBF model, the LCV model, the LGIF model and the Bregman-LBF model with different initial positions. The red contour is the initial contour, while the green contour is the segmentation results. Columns (a), (b), (c), (d) are the segmentation results of the LBF model, the LCV model, the LGIF model and the Bregman-LBF model, respectively.

\section{Experimental Results}

We applied the Bregman-LBF model on various images, and the segmentation results are presented and compared. We also conducted comparison works against the existing models: the LBF model, the LCV model and the LGIF model. There are parameters $\lambda_{1}, \lambda_{2}, \alpha, \beta, \mu$ and $v$ in the Bregman-LBF model, and the time step $\Delta t=0.1$ for the implementation. In our experiments, we note that the Bregman-LBF model is not sensitive to the choice of $\lambda_{1}, \lambda_{2}$ and $v$, thus we fixed them as $\lambda_{1}=1$, $\lambda_{2}=1, \alpha=1.05, \beta=0.95$ and $v=1$. The parameter $\mu$ of the length constraint term is changed with the images to be segmented. The weight factor $w$ is decided by the image's quality, because the global energy term can effectively segmen$\mathrm{t}$ the piece-wise image, while the local energy term is utilized to cope with the inhomogeneity image segmentation problem. In order to improve the quality of the segmentation results, we should be careful in choosing an appropriate weight factor for images in different quality.
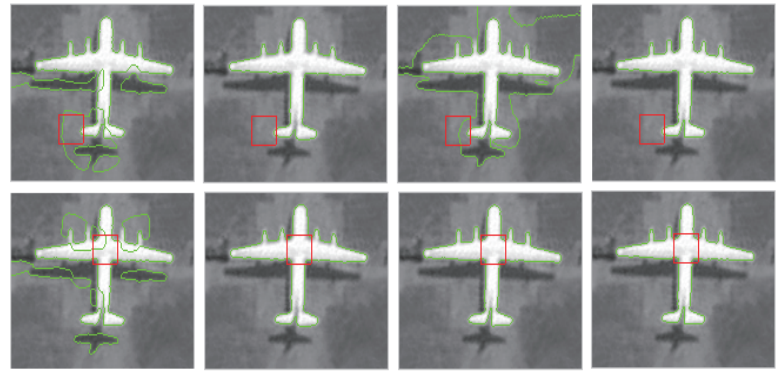

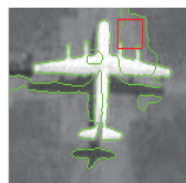

(a)

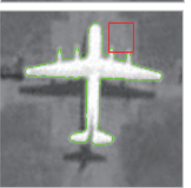

(b)

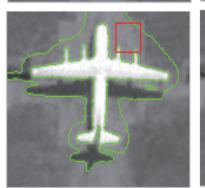

(c)

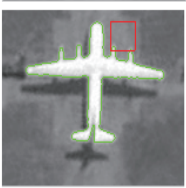

FIGURE 4. Segmentation Results of the LBF model, the LCV model, the LGIF model and the Bregman-LBF model with different initial positions. The red contour is the initial contour, while the green contour is the segmentation results. Columns (a), (b), (c), (d) are the segmentation results of the LBF model, the LCV model, the LGIF model and the Bregman-LBF model, respectively.

3.1. Robustness to Gaussian noise and intensity inhomogeneity

In order to demonstrate the robustness of our proposed model to Gaussian noise and intensity inhomogeneity, the compared results are shown in Fig.1, the first row is the segmentation results of noiseless intensity inhomogeneity image by the LBF 
model, the LCV model, the LGIF model and the proposed Bregman-LBF model, where the red contour is the initional contour and the green is segmentation results, from the result we can demonstrate that the Bregman-LBF model keep the capability of dealing with intensity inhomogeneity. The 2nd-5th rows of Fig. 1 are the segmentation results of different level of Gaussian noise with the same initial contour(the red contour), it is obvious that the Bregman-LBF model produces the best segmentation results, also demonstrate the proposed model is robust for the Gaussian noise. Moreover, we can observe that the heavier of the level of noise has, the bigger of the number of iteration is needed. These results demostrate the robustness of the Bregman-LBF model to Gaussian noise and intensity inhomogeneity.

\subsection{Robustness to contour initialization}

To demonstrate the robustness of our proposed model to contour initialization, experiments are preformed, respectively, on a synthetic image and two medical images which are typical images with intensity inhomogeneity and a real world image which almost is piece-wise, as shown in Fig.2. The different initial contours are the red contour in every image, the corresponding results which is green contour in each image of the LBF model, the LCV model, the LGIF model and the BregmanLBF model are shown in column (a),(b), (c), (d), respectively.

From the results shown in Fig.2, we can conclude that the LCV model and the LGIF model totally failed to correctly segment the object in three different initial contours. Compared with the LCV and LGIF model, the result given by the LBF model shown in column (a) shows better performance in some cases. However, we observe the Bregman-LBF model's segmentation results which shown in column (d), the BregmanLBF model can successfully extract the boundaries for three different initial contours.

From the results shown in column (a) and (c) in Fig.3, we can conclude that the LBF model and the LGIF model demonstrate certain capability of handling the intensity inhomogeneity, but the results also adequately show that these two models are sensitive to the initial placement of the level set. In other words, for these two models, if a better initial placement is chosen, satisfactory segmentation results can be obtained, or the models will be stucked in a local solution which does not satisfy the application. The LCV model can obtain almost the same segmentation results with various initial contours, which demonstrates the global property of the LCV model. However, the segmentation of the Bregman-LBF model shows that it is more robust to initial position of the contour and effective in coping with the intensity inhomogeneity.
In Fig.4, the segmentation results of the LCV model are better than those of the LBF model and the LIGF model, and are also comparable with the results of the Bregman-LBF model. The results demonstrate the inference, which the LCV model is a CV model to some extent, pointed out by [6]. The results shown in column (b) also demonstrate that the LBF model is not a good model to segment the almost piece-wise image. The LGIF model which is a combination of the CV model and the LBF model is not appropriate too, to some degree. It is obvious that the Bregman-LBF model outperforms the LBF model, LCV model and the LGIF model in this case where the images are the almost piece-wise.

Overall, we can conclude from the results in Fig.2-4 that, for various initial placements and intensity inhomogeneity images or almost piece-wise constant image, the comparing models LBF, LCV and LGIF, in some cases, can offer better segmentation results which are comparable with that of the BregmanLBF model. However, the results also show that these compared models are unstable to the initial position. If a proper initial position is provided, a good result can be obtained, otherwise unsatisfied segmentation results will be produced. The newly proposed model can correctly address the issues that we mentioned in the beginning of this paper. It has been demonstrated that the stability of the Bregman-LBF model is better than the other comparing models and the Bregman-LBF has the fine properties of both the global information based model and the local information based model.

\section{Conclusion}

In this paper, we proposed an efficient region-based active contour model for image segmentation in a variational level set framework. In our proposed model, the Bregman divergence incorporated with local binary fitting (Bregman-LBF) takes considerations of both the global and the local information to formulate the energy function in order to control the contour evolution. The global information is utilized in a way to boost its robustness to the initial placement and to accelerate the contour evolution, resulting in improving the segmentation results and reducing overall computational cost. The local information is utilized to improve the capability of handling the intensity inhomogeneity. Theoretically, we have proven that some models, e.g. the traditional Chan-Vese model, are just special cases of our proposed Bregman-LBF.Empirically, we have shown that our proposed model performs better than the existing methods in addressing the issues of intensity inhomogeneity and the robustness. 
Proceedings of the 2017 International Conference on Machine Learning and Cybernetics, Ningbo, China, 9-12 July, 2017

\section{References}

[1] L.M. Bregman. The relaxation method of finding the common point of convex sets and its application to the solution of problems in convex programming. USSR Computational Mathematics and Mathematical Physics, 7(3):200-217, 1967.

[2] T.F. Chan and L.A. Vese. Active contours without edges. Image Processing, IEEE transactions on, 10(2):266-277, 2001.

[3] M. Kass, A. Witkin, and D. Terzopoulos. Snakes: Active contour models. International Journal of Computer Vision, 1(4):321-331, 1988.

[4] C. Li, C.Y. Kao, J.C. Gore, and Z. Ding. Minimization of region-scalable fitting energy for image segmentation. Image Processing, IEEE Transactions on, 17(10):19401949, 2008.

[5] C. Li, C. Xu, C. Gui, and M.D. Fox. Level set evolution without re-initialization: a new variational formulation. In Computer Vision and Pattern Recognition.IEEE Computer Society Conference on, 1:430-436, June 2005.

[6] S. Liu and Y. Peng. A local region-based chan and vese model for image segmentation. Pattern Recognition, 45(7):2769-2779, 2012.

[7] D. Mumford and J. Shah. Optimal approximations by piecewise smooth functions and associated variational problems. Communications on Pure and Applied Mathematics, 42(5):577-685, 1989.

[8] S. Osher and J.A. Sethian. Fronts propagating with curvature-dependent speed: algorithms based on hamilton-jacobi formulations. Journal of Computational Physics, 79(1):12-49, 1988.

[9] N. Paragios and R. Deriche. Geodesic active regions and level set methods for supervised texture segmentation. In- ternational Journal of Computer Vision, 46(3):223-247, 2002.

[10] G. Paul and I.F. Cardinale, J.and Sbalzarini. Coupling image restoration and segmentation:a generalized linear model/bregman perspective. International Journal of Computer Vision, 104(1):69-93, 2013.

[11] A. Tsai, A. Yezzi Jr, and A.S. Willsky. Curve evolution implementation of the mumford-shah functional for image segmentation, denoising, interpolation, and magnification. Image Processing, IEEE Transactions on, 79(1):12-49, 2001.

[12] L.A. Vese and T.F. Chan. A multiphase level set framework for image segmentation using the mumford and shah model. International Journal of Computer Vision, 50(3):271-293, 2002.

[13] L. Wang, L. He, A. Mishra, and C. Li. Active contours driven by local gaussian distribution fitting energy. Signal Processing, 89(12):2435-2447, 2009.

[14] LingFeng Wang, Zeyun Yu, and ChunHong Pan. Medical image segmentation based on novel local order energy. In Computer Vision-ACCV 2010, pages 148-159. Springer, 2011.

[15] X.F. Wang, D.S. Huang, and H. Xu. An efficient local chan-vese model for image segmentation. Pattern Recognition, 43(3):603-618, 2010.

[16] B. Wu and Y. Yang. Local-and global-statistics-based active contour model for image segmentation. Mathematical Problems in Engineering, 2012, 2012.

[17] K. Zhang, H. Song, and L. Zhang. Active contours driven by local image fitting energy. Pattern Recognition, 43(4):1199-1206, 2010. 